\title{
Oral contraceptives use reduces ovarian and endometrial cancer risk
}

\author{
Teodora Gabriela CIOROBA ${ }^{1}$, Anca Marina CIOBANU ${ }^{1,2}$, Brindusa Ana CIMPOCA-RAPTIS ${ }^{1}$, \\ Corina GICA ${ }^{1}$, Radu BOTEZATU ${ }^{1,2}$, Gheorghe PELTECU ${ }^{1,2}$, Nicolae GICA ${ }^{1,2}$, \\ Anca Maria PANAITESCU ${ }^{1,2}$ \\ ${ }^{1}$ Filantropia Clinical Hospital, Bucharest, Romania \\ 2"Carol Davila” University of Medicine and Pharmacy, Bucharest, Romania
}

\section{ABSTRACT-}

One of the greatest problems that patients and medical system confront worldwide nowadays is cancer. Even if there is a great focus on treatment improvement, it is equally or even more important to find cancer prevention pathways. If we concentrate on gynecological malignancies, we have as example cervical cancer which can now be prevented through vaccination, but less is known about prevention methods for endometrial and ovarian cancer. In 1992, Alice S. Whittemore first described the protective role of combined oral contraceptives (COC) use against ovarian cancer. After that, many other studies tried to find out how combined oral contraceptive use influence the risk of gynecological cancers. According to all researches, it is now known that COC are associated with risk reduction in ovarian and endometrial cancers. It was observed that their risk reduction depends on different factors such as length of use, the period of life when they were used, or some other associated risk factors. Moreover, it seems that they offer protection only against some histological types of endometrial and ovarian cancer.

This review relates the most important findings regarding COC use and their risk reduction effect on ovarian and endometrial cancer in the recent studies. Even if some certain conclusions were made, such as time-dependent effect, more studies need to be conducted in order to have certain future recommendations.
\end{abstract}

Keywords: ovarian cancer, endometrial cancer, combined oral contraceptives

\section{INTRODUCTION}

Combined oral contraceptives are in use for a long time. Over time, it was observed that their use could have different benefits or on the contrary, certain disadvantages. What was important to be observed, is the way oral contraceptives influence the risk for different types of cancer. Even if most studies focused on the relation to gynecological cancers, it seems that combined oral contraceptives could also influence the risk of non-gynecological cancers.

Studies concluded that oral contraceptives reduce the ovarian and endometrial cancer risk. Along with this observation many other questions have arisen. Is this risk reduction effect dependent on the time the oral contraceptives were used, or does this benefit disappear after ceasing their administration? Might $\mathrm{COC}$ reduce the risk for other types of neoplasm? Does this benefit bring along some other risks? Is the risk reduction greater in patients having certain characteristics? Could $\mathrm{COC}$ be used as a means of prophylaxis in females with greater risk of ovarian or endometrial cancer? Is this benefit dependent on the type of oral contraceptive that is used? Is the impact different according to the histopathological type of the cancer? Are patients sufficiently informed about this benefit? This re- 
view aimed to find in literature answers for all these questions.

\section{METHODS}

PubMed database was searched for studies published from January 2014 to 2021, written in English, that analyzed the impact of $\mathrm{COC}$ use on cancer risk reduction, particularly on ovarian and endometrial risk reduction.

\section{BACKGROUND}

On the first place, as incidence, among gynecologic cancers in high-income countries and on second place worldwide [1], endometrial cancer is typically diagnosed in women between 60 and 70 years old [2]. Endometrial cancers are classified into two major types that have different risk factors and clinicopathologic features. Type 1 endometrial cancer comprises $80 \%$ of cases, while type 2 neoplasms are less common. Longterm exposure to increased estrogen levels without adequate progestin balance is known to be the primary risk factor for type 1 endometrial cancer. Few causes of unopposed estrogen excess are represented by chronic anovulation, obesity, advanced liver disease, early menarche and late menopause, infertility and nulliparity.

Ovarian cancer is the third most common gynecologic cancer in high-income countries after endometrial and cervical cancer, with a median age at diagnosis of 63 years. The lifetime risk of developing ovarian cancer is approximately $1,4 \%$. Though, the risk increases up to $60 \%$ in BRAC1 mutation carriers and up to $40 \%$ in BRCA2 mutation carriers, making the genetic mutations be the most important risk factors for ovarian cancer development. Other risk factors for ovarian cancer are older age, early menarche, late menopause, nulliparity, endometriosis or pelvic irradiation.

For both types of cancer, it was proven that $\mathrm{COC}$ administration is a protective factor. It was first mentioned in 1992 in an article published by Whittemore AS et al. that the biological changes induced by $\mathrm{COC}$ use seem to have a protective effect against ovarian cancer [3].

Probably, the chemoprotective effects of $\mathrm{COC}$ on endometrial cancer are due to the progesterone component. In the absence of a progestogen, estrogens stimulate proliferation of endometrial cells, but when progestogens are present, they induce terminal differentiation in endometrial cells, and in this way stop endometrial proliferation and prevent the neoplastic transformation. The reasons why $\mathrm{COC}$ have also a protective role on ovarian cancer are different than the ones previously described. Oral contraceptives might act directly through gonadotrophins and androgen reduction, through an apoptotic effect of synthetic pro- gestogens or through suppression of the physical effect of ovulation itself, leading to a reduction in exposure of the ovarian/fallopian tube epithelium to inflammatory or hormonal factors that could induce neoplastic transformation. Moreover, oral contraceptives reduce retrograde menstruation, which is known to be a mechanism involved in ovarian carcinogenesis [4].

\section{STUDIES RESULTS}

Not only the risk for gynecological cancers in relation with oral contraceptives was analyzed over time. A study conducted by Iversen et al. followed for up to 44 years the evolution of 46,022 women who were recruited to the UK Royal College of General Practitioners Oral Contraception Study in 1968 and 1969. According to their findings, oral contraceptives use was associated not only with reduced incidence of endometrial and ovarian cancer, but also with reduced colorectal, lymphatic, and hematopoietic cancer risk. In contrast, the study found an increased risk for breast and cervical cancer, mentioning that 5 years after oral contraceptive has stopped, this effect disappears. Another important observation was that the lung cancer risk was increased in oral contraceptives users, that were also smokers. Comparing these observations, this study concluded that choosing oral contraceptive use, most women would not be exposed themselves to long-term cancer risk, but instead, many of them might gain some protection against endometrial and ovarian cancer [5].

Karlsson et al. conducted an observational study including 256,661 women from UK Biobank in order to analyze whether between long-term oral contraceptive use and cancer risk reduction is a time dependent effect. Their results indicate that the risk of ovarian and endometrial cancer is lower among ever users, with a persistence of protective effect of oral contraceptive up to 30-35 years after administration discontinuation. Though, they observe a slightly different impact regarding the period length of oral contraceptive use and the two types of cancer. It seems that the risk reduces with longer duration of use, especially for endometrial cancer. Regarding ovarian cancer, they observed that after 10 years of oral contraceptive administration, the risk does not decrease additionally. Interestingly, regarding the concern that oral contraceptive use increases the risk for breast cancer, this study observed that only during the first 2 years after discontinuation, the risk for breast cancer was higher. That is why we could consider that the dramatic reduction of ovarian and endometrial cancer outweighs the breast cancer risk. Patients should know all the pros and cons in order to make an informed decision [6].

Not only the duration of contraceptive use, but also the exact moment in life when they have been used 
seems to have an importance regarding the degree of protection. One analysis based on four studies coordinated by University of Southern California, included 1,632 cases of women with ovarian cancer and 2,340 controls to find out if such relation exists. One of the conclusions was that the protection of combined oral contraceptives per year against ovarian cancer would be greater if the pills were used before age 35 than the use after this age [7].

Regarding the moment of life when the oral contraceptives were used and the risk reduction of ovarian cancer, it was also searched if there is any relation between their use and the time of full-term pregnancies. One case-control study including Canadian population discovered that the ovarian cancer risk reduction in parous women is approximate $40 \%$ if the COC were administered exclusively before the first full-term pregnancy (FFTP). This grade of risk reduction tends to be almost as high as the risk reduction observed in women who used $\mathrm{COC}$, both before and after FFTP, which is $50 \%$. What is also an interesting finding in this study is that also short-term use of COC before the first FFTP, that means less than 5 years, brings a consistent reduction in future risk of ovarian cancer. These findings suggests that the use of COC before the FFTP might be used as a prevention strategy considering the persistent ovarian cancer risk reduction. The physio-pathological mechanism that could explain this strong lasting impact of COC use before the FFTP on ovarian cancer risk reduction is yet unknown [8].

In Denmark, two different studies analyzed the impact of hormonal contraception on ovarian, respectively endometrial cancer.

The first study, published in 2018 by Iversen et al., included all women living in Denmark aged $15-49$ years, between 1 January 1995 and 31 December 2014, that means a follow up comprising a total of 1,879,227 women, over a period of 20 years. The result showed that $21 \%$ of ovarian cancers were prevented by contraceptives administration. However, the protective effect was not confirmed for progesterone only pills, but only for $\mathrm{COC}$, no matter the types of progestogens they contain. This study analyzed also if the impact is different dependent on the histopathological type of ovarian cancer. The risk was significantly reduced for endometrioid, mucinous and serous epithelial cancer, but the reduction was not significant also for clear cell epithelial and non-epithelial ovarian cancer [9].

The other study conducted in Denmark by Iversen et al. analyzed the effect of oral contraceptives on endometrial cancer. The data estimated that $25 \%$ of endometrial cancer were prevented in the population who used hormonal contraception. Similar to the previous described study, progestin-only pills did not lower the risk for endometrial cancer. Again, it was analyzed if cer- tain histopathological types of endometrial cancer might benefit more from oral contraceptives administration. The results indicate that the risk is almost halved for type one endometrial carcinoma but might be increased for the rare cases of endometrial sarcomas [10].

In accordance with the previous mentioned study, a meta-analysis of 36 epidemiological studies confirmed that the effect of oral contraceptives might vary by histological subtype, with a strongly association between ever users and reduced risk of type I and probably type II endometrial cancer, but without such association with uterine sarcomas. This metanalysis performed by the Collaborative Group on Epidemiological Studies on Endometrial Cancer also confirmed that the risk for endometrial cancer is reduced by current or past administration of oral contraceptives, being greater with the longer use. Objectively, their results showed that during a period of 50 years (1965-2014), 400,000 cases of endometrial cancer were prevented by oral contraceptives, including only in the last 10 years of this period 200,000 prevented cases. An important observation of this meta-analysis was that the beneficial effect appeared no matter the quantity of estrogen contained in the combined contraceptive pills. This observation was possible because during 1960-1980 the pills had higher estrogen doses in comparison with the new one [11].

Even if all the previous mentioned studies confirmed that the beneficial effect of COC on ovarian and endometrial cancer risk reduction is greater with the duration of use, the question is whether there are other lifestyle factors that might influence this effect. One prospective study conducted by Michels et al. including 196,536 women between 50 and 71 years old, identified a greater risk reduction for endometrial cancer among long-term users who were also smokers, were obese, with a BMI of 30 or greater. The same observation was made for women consuming alcohol or having a sedentary lifestyle. A reduction by $50 \%$ to $60 \%$ was registered among women who exercised moderately or infrequently if they used oral contraceptives in the long run. Analyzing if the same effect is observed for ovarian cancer across these modified lifestyle factors, the study concluded that the reduction is consistent no matter how these factors vary [12]. Having this information, we ask ourselves if oral contraceptives might be beneficial for chemoprevention in women having risk factors that influence their baseline cancer risk.

Apart from all these observations, investigators wanted to know how oral contraceptive use will influence the risk for endometrial and ovarian cancer in women with genetic mutations known to significantly increase the risk for these types of cancers. Mainly, there were observed the effects in women having BRCA mutations or Lynch syndrome. 
A review by Huber et al. included 4 meta-analyses and 1 systematic review regarding ovarian and breast cancer risk, one case-control study and one retrospective cohort study on ovarian cancer risk, one case-control, 2 case-only, one prospective and one retrospective cohort study, on oral contraceptive induced breast cancer risk in BRCA mutation carriers. The conclusion was that oral contraceptive use in BRCA mutation carriers reduce the risk for ovarian cancer. However, for these patients, cannot be excluded an increase in breast cancer risk. Therefore, carriers of BRCA1/2 mutations must be informed that oral contraceptive use may increase the risk for breast cancer and that they should not be used for the prevention of ovarian cancer [13].

Davidson et al. performed a review in order to analyze how oral contraceptive administration influence patients with genetic susceptibilities due to mutations in BRCA1, BRCA2 or genes involved in Lynch syndrome. Their observations suggested that the use of oral contraceptive might be taken into consideration for women with Lynch syndrome, given the possibility of a risk reduction for the syndrome associated malignancies, including endometrial and ovarian cancer. From their study it also appears that in BRCA1/2 mutation carriers the ovarian cancer risk is reduced at least as great as reported in the general population. Still, the potential of increasing the breast cancer risk, in particular for these patients, is a warning that chemoprevention with oral contraceptive for ovarian cancer is less willing in BRCA1/2 mutations carriers [14].

Seeing all the benefits that oral contraceptives use might bring regarding endometrial and ovarian cancer risk reduction, we also researched if doctors are up to date with this information and if patients have any knowledge about these benefits.

A cross-section study was carried out in Italy in 2015 by Nappi et al. in order to investigate levels of knowledge that educated women aged between 18 and 44 years have about combined hormonal contraceptive and their impact on wellbeing. It was interesting to note that $67 \%$ participants knew that combined oral contraceptives have a protective effect on ovarian cancer and $53 \%$ were aware of the protective effect on endometrial cancer [15].
In Virginia, Horst et al. assessed how family physicians and obstetrician/gynecologists prescribe and counsel females regarding oral contraceptives and cancer prevention. The survey showed that gynecologists were more likely than family physicians to cite hormonal contraceptives as indication for endometrial cancer prevention. Prescription of hormonal contraception for endometrial or ovarian cancer prevention was identified in less the $2 \%$ of cases, also gynecologist being more likely to prescribe these pills for those indications. The study concluded that improvements in knowledge about cancer risk and the use of oral contraceptives as chemoprevention need to be done in order to optimize primary ovarian and endometrial cancer prevention [16].

\section{CONCLUSIONS}

Combined oral contraceptives use is associated with risk reduction of both ovarian and endometrial cancer. Moreover, some studies also described a protective effect against colorectal, lymphatic and hematopoietic cancers. The longer the period of use, the stronger is the protective effect, especially for endometrial cancer. Protective effect against ovarian cancer seems to be stronger if $\mathrm{COC}$ are used before age 35 and also if they are used before the first full-term pregnancy. It was proven that progestin-only pills do not provide the same protective effect against ovarian and endometrial cancers. It is also possible that the protective effect depends on histopathological type of cancer, being observed especially for endometrioid, mucinous and serous epithelial ovarian cancer, respectively for type one endometrial cancer. The risk reduction effect might be amplified in women having other risk factors such as smoking, obesity, alcohol consumption, sedentary lifestyle. Although the risk reduction effect on ovarian cancer was proved among carriers of BRCA1/2 mutations, their use in these situations is still not recommended, taking into account the potential of increasing the risk for breast cancer. All previous described benefits suggest that it is important to make sure that doctors are aware of these, so that they can inform their patients and recommend COC knowing also their long-term beneficial effects.

Conflict of interest: none declared Financial support: none declared

\section{REFERENCES}

1. Bray F, Ferlay J, Soerjomataram I, Siegel RL, Torre LA, Jemal A. Global cancer statistics 2018: GLOBOCAN estimates of incidence and mortality worldwide for 36 cancers in 185 countries. CA Cancer J Clin. 2018 Nov;68(6):394-424.
2. Epidemiology of Endometrial Cancer Consortium (E2C2). Available at: https://epi. grants.cancer.gov/eecc/. 
3. Whittmore AS, Harris R, Itnyre J. Characteristics Relating to Ovarian Cancer Risk: Collaborative Analysis of 12 US Case-Control Studies. Am J Epidemiol. 1992;136(10):1184-1203.

4. Jordan SJ, Wilson LF, Nagle CM, Green AC, Olsen CM, Bain CJ, Pandeya N, Whiteman DC, Webb PM. Cancers in Australia in 2010 attributable to and prevented by the use of combined oral contraceptives. Aust N Z J Public Health. 2015 Oct;39(5):441-5.

5. Iversen L, Sivasubramaniam S, Lee AJ, Fielding S, Hannaford PC. Lifetime cancer risk and combined oral contraceptives: the Royal College of General Practitioners' Oral Contraception Study. Am J Obstet Gynecol. 2017 Jun;216(6):580.e1-580.e9.

6. Karlsson T, Johansson T, Höglund J, Ek WE, Johansson A. Time-dependent effects of oral contraceptive use on breast, ovarian and endometrial cancers. Cancer Res. 2020 Dec 17:canres.2476.2020.

7. Wu AH, Pearce CL, Lee AW, Tseng C, Jotwani A, Patel P, Pike MC. Timing of births and oral contraceptive use influences ovarian cancer risk. Int J Cancer. 2017 Dec 15;141(12):2392-2399.
8. Cook LS, Pestak CR, Leung AC, Steed H, Nation J, Swenerton K, Gallagher R, Magliocco A, Köbel M, Brooks-Wilson A, Le N. Combined oral contraceptive use before the first birth and epithelial ovarian cancer risk. $\mathrm{Br}$ J Cancer. 2017 Jan 17;116(2):265-269.

9. Iversen L, Fielding S, Lidegaard $\varnothing$, Mørch LS, Skovlund CW, Hannaford PC. Association between contemporary hormonal contraception and ovarian cancer in women of reproductive age in Denmark: prospective, nationwide cohort study. BMJ. 2018 Sep 26;362:k3609.

10. Iversen L, Fielding S, Lidegaard $\varnothing$, Hannaford PC. Contemporary hormonal contraception and risk of endometrial cancer in women younger than age 50: A retrospective cohort study of Danish women. Contraception. 2020 Sep;102(3):152-158.

11. Collaborative Group on Epidemiological Studies on Endometrial Cancer. Endometrial cancer and oral contraceptives: an individual participant meta-analysis of 27276 women with endometrial cancer from 36 epidemiological studies. Lancet Oncol. 2015 Sep;16(9):1061-1070.

12. Michels KA, Pfeiffer RM, Brinton LA, Trabert B. Modification of the Associations Between
Duration of Oral Contraceptive Use and Ovarian, Endometrial, Breast, and Colorectal Cancers. JAMA Oncol. 2018 Apr 1;4(4):516521.

13. Huber D, Seitz S, Kast K, Emons G, Ortmann O. Use of oral contraceptives in BRCA mutation carriers and risk for ovarian and breast cancer: a systematic review. Arch Gynecol Obstet. 2020 Apr;301(4):875884.

14. Davidson BA, Moorman PG. Risk-benefit assessment of the combined oral contraceptive pill in women with a family history of female cancer. Expert Opin Drug Saf. 2014 Oct;13(10):1375-82.

15. Nappi RE, Pellegrinelli A, Campolo F, Lanzo G, Santamaria V, Suragna A, Spinillo A, Benedetto C. Effects of combined hormonal contraception on health and wellbeing: women's knowledge in northern Italy. Eur J Contracept Reprod Health Care. 2015 Feb;20(1):36-46.

16. Horst KE, Modesitt SC. Hormonal Contraceptives for Endometrial Cancer Prevention in Obese and High-Risk Women in Virginia. South Med J. 2016 Oct;109(10):621-627. 